\title{
Efecto tóxico de $\beta$-cipermetrina, deltametrina y fenitrotión en cepas de Triatoma dimidiata (Latreille, 1811) y Triatoma maculata (Erichson, 1848) (Hemiptera, Reduviidae)
}

\author{
Marlene Reyes ${ }^{1}$, Víctor Manuel Angulo ${ }^{2}$, Claudia Magaly Sandoval ${ }^{2}$ \\ ${ }^{1}$ Escuela de Biología, Facultad de Ciencias, Universidad Industrial de Santander, Piedecuesta, Santander, \\ Colombia. \\ ${ }^{2}$ Centro de Investigaciones en Enfermedades Tropicales de la Universidad Industrial de Santander, \\ CINTROP-UIS, Piedecuesta, Santander, Colombia.
}

Introducción. La evaluación de la susceptibilidad a insecticidas de las diferentes especies de triatominos, involucrados en la transmisión de la enfermedad de Chagas de cada región, es indispensable para el éxito de las campañas de control.

Objetivo. Determinar la línea base de susceptibilidad para los principios activos deltametrina, $\beta$-cipermetrina y fenitrotión en ninfas de primer y quinto estadio de Triatoma dimidiata y en ninfas de primer estadio de Triatoma maculata.

Materiales y métodos. Se utilizó el protocolo de evaluación de la actividad insecticida en triatominos (técnica de aplicación tópica) para las especies en estudio.

Resultados. Los valores de DL50 en ninfas de primer estadio de $T$. maculata expresados en nanogramos/insecto (ng/i) fueron de: 0,07, 0,05 y 4,12. Las DL99 fueron de: 1,08, 0,37 y 17,89 para deltametrina, $\beta$ - cipermetrina y fenitrotión respectivamente. En $T$. dimidiata los valores de DL 50 fueron de: 0,44, 0,46 y 16,45. Las DL99 obtenidas fueron de: 2,22, 1,97 y 36,07 ng/i para deltametrina, $\beta$ - cipermetrina y fenitrotión respectivamente. En ninfas de quinto estadio de $T$. dimidiata las DL50 fueron de: 510,72, 1623,59 y 838,91. Las DL99 fueron de: 9607,50, 11717,91 y 1525 para deltametrina, $\beta$ - cipermetrina y fenitrotión respectivamente.

Conclusión. En ninfas de primer estadio de T. dimidiata y $T$. maculata los insecticidas piretroides fueron mas efectivos; en ninfas de quinto estadio de $T$. dimidiata la efectividad de los piretroides y del organofosforado fue diferente con las DL50; las ninfas de este estadio requirieron dosis altas comparadas con las utilizadas para otros triatominos, lo cual sugiere una baja susceptibilidad. La DL99 para el organofosforado fue significativamente menor, lo que podría indicar una mayor efectividad en campo. Es importante realizar estudios de efectos sinergistas para mostrar el posible rol de mecanismos bioquímicos que determine su tolerancia a los piretroides, esto representa un nuevo reto para las campañas de control en los países andinos y centroamericanos donde esta especie es endémica.

Palabras clave: Triatominae, Triatoma, enfermedad de Chagas, insecticidas organofosforados, piretroides.

Toxic effect of $\beta$-cipermethrin, deltamethrin and fenitrothion in colonies of Triatoma dimidiata (Latreille, 1811) and Triatoma maculata (Erichson, 1848) (Hemiptera, Reduviidae)

Introduction. The susceptibility to insecticides of triatomine species must be evaluated because of their involvement in the transmission of the Chagas disease. In each region with Chagas endemicity, evaluation of insecticide response is necessary to predict the success of the control campaigns.

Objective. The baseline susceptibility was determined for the active principles deltamethrin, $\beta$ cypermethrin and fenitrothion in nymphs of first and fifth instar of Triatoma dimidiata and nymphs of first instar of Triatoma maculata.

Materials and methods. The insecticide activity in triatomines was evaluated by the technique of topical application. 
Results. The values of the LD50 in nymphs of first instar for T. maculata, expressed in nanograms per insect (ng/i), were $0.07,0.05$ and 4.12 for deltamethrin, $\beta$-cypermethrin and fenitrothion respectively. The corresponding LD99 values were $1.08,0.37$ and $17.89 \mathrm{ng} / \mathrm{i}$. In T. dimidiata, the LD50 values were $0.44,0.46$ and $16.45 \mathrm{ng} / \mathrm{i}$; the LD99 values were $2.22,1.97$ and 36.07 ng/i. In nymphs of fifth instar T. dimidiata, the LD50 values were 510.7, 1,623.6 and $838.9 \mathrm{ng} / \mathrm{i}$; the LD99 values were 9,607.5, 11,717.9 and 1,525.0 ng/i, respectively.

Conclusion. In first instar nymphs of $T$. dimidiata and $T$. maculata, the pyrethroid insecticides were more effective; in fifth instar nymphs of $T$. dimidiata, the effectiveness of the pyrethroids and the organophosphate differed in the LD50 comparison-the nymphs required much higher doses compared with the other triatomines and suggested a low susceptibility. The LD99 for the organophosphate (fenitrothion) was significantly lower and may indicate its greater effectiveness in field. Studies of synergistic effects amonst insecticides are important to clarify the role of biochemical mechanisms that determine tolerance to the pyrethroids. Insecticide tolerance represents a new challenge for control campaigns in the Andean and Central American countries where Chagas disease is endemic.

Key words: Triatominae, Triatoma, Chagas disease, insecticides, organophosphate, pyrethrins,

La enfermedad de Chagas afecta a una población de 16 a 18 millones de personas, y más de 100 millones se encuentran en riesgo de infección en 21 países (1). En Latinoamérica las especies domiciliadas más importantes implicadas en la transmisión de Trypanosoma cruzi son Triatoma infestans, en los países del Cono sur, Rhodnius prolixus, en Centro América y el Norte de Sur América, y Triatoma dimidiata, que se extiende a lo largo de la costa Pacífica desde México hasta el Ecuador y el norte de Perú (2-4). En Colombia, durante los últimos 20 años, $T$. dimidiata ha ocupado hábitats domiciliarios, peridomiciliarios, silvestres y viviendas de buena construcción en cabeceras municipales. Este fenómeno ha ocurrido también en algunas ciudades de Centroamérica (4-8).

Triatoma maculata se distribuye en la zona nororiental de Sur América, colonizando ambientes domésticos, peridomésticos, silvestres y urbanos del país con altos niveles de infestación $(2,9)$ Mojíca MT, Cuervo LA, Ariza K, Chacón E, Chacón R, Dib JC, et al. Distribución de Triatoma maculata e infestación domiciliaria en Santa Marta, Colombia. Biomédica 2003;23:96).

\footnotetext{
Correspondencia:

Marlene Reyes Jerez, Laboratorio de Entomología,

Centro de Investigaciones en Enfermedades Tropicales de la Universidad Industrial de Santander, Km 2 vía El Refugio, Piedecuesta, Santander, Colombia.

Tel: 6563971, fax: 6540177.

marejez1@hotmail.com
}

Recibido: 22/08/05; aceptado: 02/06/06
Las experiencias de control vectorial en América Central, los países Andinos y del Cono Sur han demostrado que una de las pocas alternativas prácticas para controlar la enfermedad de Chagas es a través del control de los triatominos domiciliarios $(10,11)$. En 1996 se creó en Colombia el Programa Nacional de Control Vectorial de la enfermedad de Chagas (12); en su marco se han iniciado actividades de aspersión de insecticidas piretroides y organofosforados y políticas de mejoramiento de vivienda en Boyacá, Casanare, Santander, Norte de Santander y Arauca $(12,13)$. Dichos departamentos forman parte de las zonas catalogadas como de alto y mediano riesgo de infección, donde el uso de la deltametrina se ha hecho extensivo como medida de control (14).

Algunos países de Centroamérica han tenido éxito utilizando piretroides en la eliminación de colonias de $T$. dimidiata intradomiciliarias (15-7). Sin embargo, las zonas infestadas con triatominos de algunos países de Suramérica han mostrado diferencias en la susceptibilidad y la resistencia a insecticidas en poblaciones de $T$. infestans de Brasil y Argentina, así como en una población de R. prolixus en Carabobo, Venezuela (18-21). En Colombia, estudios recientes muestran altos niveles de infestación postratamiento; una de las causas sugeridas por los investigadores es la presencia de poblaciones de esta especie con menor susceptibilidad al piretroide usado (5).

Teniendo en cuenta que en la actual situación colombiana una de las estrategias que hacen parte 
del control integrado de los triatominos es el uso de sustancias químicas insecticidas, se hace necesario evaluar el efecto triatomicida en el laboratorio con el fin de monitorizar la susceptibilidad y la resistencia de las poblaciones blanco a los insecticidas en uso.

En el país son pocos los estudios sobre este tema que se reportan en la literatura, y todos han estado dirigidos a evaluar la susceptibilidad y resistencia en $R$. prolixus $(22,23)$.

Si el conocimiento de $T$. dimidiata en este aspecto es escaso, también lo es en cuanto a la determinación de factores que influyen en la respuesta a los insecticidas como por ejemplo el estado nutricional; las observaciones sobre la actividad insecticida con $\beta$-cipermetrina aplicada por vía tópica en ninfas de $V$ estadio muestran diferencias que indican una muy baja absorción insecticida asociada con mecanismos de detoxificación (datos no publicados, CINTROP-UIS).

En este estudio se propuso establecer la línea de base de susceptibilidad en $T$. dimidiata y $T$. maculata con dos piretroides y un organofosforado como inicio de los estudios de monitorización de la susceptibilidad y la resistencia a insecticidas en poblaciones de campo, ante todo para orientar las políticas de control en el país y, además, para contribuir a la extensión de los protocolos de evaluación de la actividad insecticida de los triatominos en Latinoamérica.

\section{Materiales y métodos}

\section{Material biológico}

Se utilizaron cepas de $T$. dimidiata y $T$. maculata provenientes de San Joaquín y San José de Miranda, municipios del departamento de Santander, áreas éstas sin tratamiento sistemático de control estatal en el momento de la recolección. La cría de las cepas se inició en 1998, manteniéndolas sin aporte de material externo en condiciones ambientales constantes de laboratorio a $25 \pm 2^{\circ} \mathrm{C}, 70$ a $80 \%$ de HR y fotoperíodo de 12:12, y alimentándolas cada 15 días con sangre de gallina.

En los ensayos biológicos con insecticidas se utilizaron ninfas de primer estadio de $T$. dimidiata y T. maculata; las ninfas I tenían entre 24 y 36 horas (24), con un peso entre $1,4 \pm 2,1 \mathrm{mg}$ y $0,6 \pm 1,1 \mathrm{mg}$, respectivamente, y las ninfas de quinto estadio de $T$. dimidiata tenían 10 a 12 días de edad, no recibieron alimento y su peso era de $97 \pm 30 \mathrm{mg}$ (datos no publicados, CINTROP-UIS).

\section{Insecticidas}

Los insecticidas (grado técnico) utilizados en este estudio fueron deltametrina al 99,1\% (Agrevo S.A, Argentina), $\beta$-cipermetrina al 99,4\% (Chemotecnica Sintyal, Argentina), y fenitrotión al 98,5\% (Lab Dr.Ehrenstorfer-Schafers, Germany). Las diluciones seriales de insecticidas se prepararon en acetona (JT Baker, México).

\section{Ensayos biológicos}

Evaluación del efecto insecticida. Las ninfas de primer estadio de $T$. dimidiata y $T$. maculata y las de quinto estadio de $T$. dimidiata se trataron con aplicación tópica de principios activos (fenitrotión, $\beta$-cipermetrina y deltametrina) diluidos y aplicados con microjeringas Hamilton de 5 y $25 \mu$ provistas de descargador repetitivo. En la región dorsal del abdomen de cada ninfa en estadios I y $\mathrm{V}$ se aplicaron 0,1 y $0,5 \mu \mathrm{l}$ de la solución, respectiva mente. En los ensayos biológicos se seleccionaron cuatro niveles de dosis que registraron entre $10 \mathrm{y}$ $90 \%$ de mortalidad; se utilizaron 10 ninfas por dosis con mínimo tres réplicas en diferentes días; como grupo control se utilizaron 10 insectos en cada réplica con igual volumen de acetona. Después del tratamiento, los insectos se colocaron en frascos plásticos y se mantuvieron en una incubadora bajo condiciones ambientales constantes a $25 \pm 2^{\circ} \mathrm{C}, 70$ a $80 \%$ de HR. La lectura de mortalidad para el insecticida organofosforado fenitrotión se realizó a las 48 horas y para los piretroides $\beta$-cipermetrina y deltametrina a las 72 horas de aplicación del tratamiento.

Criterio de muerte. Se consideró muerto el insecto que colocado sobre un papel de filtro no tuviera actividad locomotora propia, ya fuera en forma espontánea o al ser estimulado con un pincel o una pinza según lo establecido por el protocolo de evaluación de la actividad insecticida en triatominos de la Organización Mundial de la Salud (24). 


\section{Análisis de datos}

Los valores obtenidos de la dosis-respuesta en los ensayos biológicos fueron sometidos al análisis EPA PROBIT versión 1.5 de 1999 para determinar los valores estadísticos DL50 y DL 99. Se aplicó una prueba de $\mathrm{ji}^{2}$ según el caso para comparar los niveles de significación. El potencial insecticida de la $\beta$-cipermetrina y el fenitrotión se calculó tomando como referencia la deltametrina: DL50 de deltametrina/DL50 de $\beta$-cipermetrina o fenitrotión $x 100$. Se utilizó la deltametrina como referencia por su comprobado efecto triatomicida (25)

\section{Resultados}

Mediante un análisis estadístico de los valores de DL50 por aplicación tópica de ambos piretroides en ninfas de primer estadio de $T$. dimidiata, en el cuadro 1 se indica que los parámetros DL50 de la deltametrina y la $\beta$-cipermetrina son similares $(p$ $=0,82)$ y no presentan diferencias estadísticas. Se evidenció una mayor toxicidad de la deltametrina $(p<0,05)$ y la $\beta$-cipermetrina $(p<$ $0,05)$ con diferencias significativas respecto al fenitrotión. En este estadio, la DL99 también mostró una mayor toxicidad de los piretroides deltametrina y $\beta$-cipermetrina $(p<0,05)$, con diferencias significativas respecto al organofosforado fenitrotión.

Los valores de las pendientes de los insecticidas deltametrina, $\beta$-cipermetrina y fenitrotión en ninfas de primer estadio de $T$. dimidiata indican que la respuesta de la cepa fue heterogénea para deltametrina $(3,3)$ y $\beta$-cipermetrina $(3,6)$, a diferencia de la respuesta homogénea $(6,8)$ del fenitrotión $(p<$ $0,05)$ que sí presentó diferencias estadísticas.

Los valores obtenidos de DL50 en ninfas de primer estadio de $T$. maculata (cuadro 1) muestran un efecto tóxico similar de la $\beta$-cipermetrina y la deltametrina $(p=0,34)$, sin presentar diferencias significativas. Los valores de DL50 y DL99 obtenidos en ambos piretroides indican $(p<0,05)$ que son significativamente menores (mayor toxicidad) que los determinados en fenitrotión.

Los valores de las pendientes en ninfas de primer estadio de $T$. maculata para deltametrina $(1,9)$ y $\beta$-cipermetrina $(2,7)$ sugieren que la respuesta es heterogénea para los insecticidas piretroides comparados con el fenitrotión $(3,6)(p<0,05)$, presentando diferencias significativas.

Los valores DL 50 y DL 99 por aplicación tópica en ninfas de quinto estadio de $T$. dimidiata de los tres insecticidas se muestran en el cuadro 1. Es sorprendente la baja toxicidad de los insecticidas estudiados en ninfas $V$ de esta especie. En este contexto de marcada tolerancia, y tomando como parámetro la DL50, se observa que la deltametrina y el fenitrotión son más activos que la $\beta$-cipermetrina $(p<0,05)$ con diferencias significativas. Con el parámetro estadístico DL99 encontramos mayor efectividad del fenitrotión frente a los piretroides $(p<0,05)$, siendo la diferencia significativa.

Cuadro 1. Nivel de susceptibilidad en ninfas de estadios I y V sin alimentar de Triatoma dimidiata y Triatoma maculata, cepa referencial susceptible a la aplicación tópica de los principios activos deltametrina, $\beta$-cipermetrina y fenitrotión.

\begin{tabular}{|c|c|c|c|c|c|c|c|c|}
\hline Especie & Estadio & Insecticida & DL50 ng/i & IC 95\% & DL99ng/i & IC95\% & $\mathrm{b} \pm \mathrm{DE}$ & $\mathbf{n}$ \\
\hline \multirow[t]{6}{*}{ Triatoma dimidiata } & I & deltametrina & 0,44 & $0,38-0,51$ & 2,22 & $1,69-3,75$ & $3,3 \pm 0,4$ & 240 \\
\hline & I & $\beta$-cipermetrina & 0,46 & $0,39-0,52$ & 1,97 & $1,42-3,44$ & $3,6 \pm 0,8$ & 250 \\
\hline & I & fenitrotión & 16,45 & $15,22-17,64$ & 36,07 & $31,24-44,86$ & $6,8 \pm 0,5$ & 200 \\
\hline & V & deltametrina & 510,72 & $279,49-706,23$ & 9607,50 & $3819,48-170498$ & $1,8 \pm 0,4$ & 180 \\
\hline & V & $\beta$-cipermetrina & 1623,59 & $1322,36-1894,34$ & 11717,91 & $6767,58-42048,42$ & $2,7 \pm 0,5$ & 200 \\
\hline & V & fenitrotión & 838,91 & $791,36-887,51$ & 1525 & $1277,69-2302,04$ & $8,9 \pm 1,8$ & 220 \\
\hline \multirow[t]{3}{*}{ Triatoma maculata } & I & deltametrina & 0,07 & $0,05-0,09$ & 1,08 & $0,46-7,67$ & $1,9 \pm 0,5$ & 102 \\
\hline & I & $\beta$-cipermetrina & 0,05 & $0,05-0,07$ & 0,37 & $0,21-1,45$ & $2,7 \pm 0,4$ & 151 \\
\hline & I & fenitrotión & 4,12 & $3,56-4,78$ & 17,89 & $11,89-41,64$ & $3,6 \pm 0,7$ & 150 \\
\hline
\end{tabular}

DL50 = dosis que ocasiona el $50 \%$ de mortalidad de los insectos expuestos, expresada en nanogramos por insecto; DL99 = dosis que ocasiona el $99 \%$ de la mortalidad de los insectos expuestos, expresada en nanogramos por insecto. $\mathrm{b}=$ pendiente de la recta; $\mathrm{DE}=$ desviación estándar; $\mathrm{n}=$ número total de insectos. 
En ninfas de quinto estadio de $T$. dimidiata, los valores de las pendientes encontrados (cuadro 1) muestran que la respuesta de la población fue heterogénea para la deltametrina $(1,8)$ y para la $\beta$ cipermetrina $(2,7)$. En cambio se observó una respuesta homogénea $(8,9)$ para el fenitrotión con diferencias significativas $(p<0,05)$ respecto a los piretroides.

La efectividad insecticida, medida por el potencial insecticida, se muestra en el cuadro 2. En ninfas de primer estadio de $T$. dimidiata, la toxicidad intrínseca de la deltametrina fue similar a la de la $\beta$-cipermetrina, pues sólo fue 1,02 veces más efectiva, pero respecto al fenitrotión mostró una efectividad 37,1 veces mayor. En ninfas de quinto estadio, y en el marco de la acentuada tolerancia observada a los insecticidas estudiados, la toxicidad intrínseca de la deltametrina sólo fue 3,2 veces mayor que la de la $\beta$-cipermetrina y 1,6 veces mayor que la del fenitrotión. En ninfas I de T. maculata, la $\beta$-cipermetrina presentó 1,2 veces más potencial insecticida que la deltametrina; la deltametrina fue 63 veces más efectiva que el fenitrotión.

\section{Discusión}

El análisis de los valores de DL50 obtenidos para cada insecticida en ninfas de primer estadio de $T$. dimidiata y $T$. maculata muestra claramente las diferencias de efectividad entre los insecticidas piretroides y el organofosforado en estudio, lo que se asemeja a lo reportado en ninfas I de $T$. infestans susceptibles (deltametrina $0,13 \mathrm{ng} / \mathrm{i} ; \beta$ cipermetrina $0,24 \mathrm{ng} / \mathrm{i}$, y fenitrotión 21,6 ng/i) (20), y concuerda con la reconocida acción triatomicida de los piretroides (25). Todos los insecticidas mostraron mayor toxicidad en ninfas de primer estadio que en ninfas de quinto estadio; estas diferencias de toxicidad pueden ser atribuidas a diferencias en los procesos de toxicocinética en cada uno de los estadios (26). Considerando las dos especies y todos los insecticidas estudiados en ninfas de primer estadio, T. maculata es más susceptible que $T$. dimidiata en cuanto a los parámetros de toxicidad expresados en peso de ingrediente activo por insecticida con relación al peso del insecto vivo; esta condición fue observada por Oliveira Filho para $R$. prolixus al compararlo con $T$. infestans y Panstrongylus megistus (21).

Los resultados obtenidos en ninfas de quinto estadio de $T$. dimidiata mostraron su sorprendente tolerancia a los insecticidas estudiados, particularmente a la deltametrina, aunque si bien éste fue el insecticida más efectivo, las dosis necesarias para causar volteo al $50 \%$ de los insectos tratados fueron altas en comparación con las requeridas en otras especies (27). En este

Cuadro 2. Potencial insecticida por aplicación tópica de la $\beta$-cipermetrina y fenitrotión versus deltametrina en ninfas I de Triatoma dimidiata y Triatoma maculata y ninfa $\mathrm{V}$ de Triatoma dimidiata.

\begin{tabular}{lccc}
\hline Especie & Insecticida & Estadio & Potencial insecticida \\
\hline Triatoma dimidiata & deltametrina & $\mathrm{I}$ & 100 \\
& $\beta$-cipermetrina & $\mathrm{I}$ & 98,31 \\
& fenitrotión & $\mathrm{I}$ & 2,69 \\
& deltametrina & $\mathrm{V}$ & 60,87 \\
& fenitrotión & $\mathrm{V}$ & 31,45 \\
Triatoma maculata & $\beta$-cipermetrina & $\mathrm{I}$ & 100 \\
& deltametrina & $\mathrm{I}$ & 122 \\
& $\beta$-cipermetrina & $\mathrm{I}$ & 1,57 \\
\hline
\end{tabular}

Potencial insecticida $=\frac{\text { DL50 o CL } 50 \text { deltametrina }}{\text { DL50 o CL50 } \beta \text {-cipermetrina o fenitrotión }} \times 100$
DL: dosis letal
CL: concentración letal


estudio se reportó una DL50 de 510 ng/i para esta especie; con $T$. infestans se presentó una DL50 de $215 \mathrm{ng} / \mathrm{i}$ (25). Es posible plantearse la pregunta sobre qué mecanismos enzimáticos con rol clave en la detoxificación de insecticidas, particularmente de los piretroides, podrían tener una actividad marcada en ninfas $\mathrm{V}$ de $T$. dimidiata como para conferirles tolerancia frente a la acción triatomicida.

Al comparar los valores estadísticos DL50 1623,59 $\mathrm{ng} / \mathrm{i}$ en ninfas $\mathrm{V}$ de $T$. dimidiata sin alimentar con los DL50 432,57 ng/i en ninfas $V$ alimentadas se observa una menor respuesta tóxica a la $\beta$ cipermetrina en las ninfas de $\mathrm{V}$ estadio sin alimentar que en las alimentadas, lo cual evidencia mayor susceptibilidad de las ninfas $V$ alimentadas y sugiere una baja absorción de este insecticida en ninfas sin alimentar. Este hallazgo es similar a lo reportado en ninfas de segundo estadio de $T$. infestans, en las cuales se encontró un importante incremento en la penetración y mortalidad con DDT en insectos tratados después de la alimentación (28); estas diferencias de penetración en ninfas sin alimentar sugieren baja absorción del insecticida, lo que asociado a los caminos metabólicos de desintoxicación ya establecidos, podrían explicar la muy baja actividad del insecticida.

Una población de insectos susceptible a insecticidas presenta valores elevados de pendiente (población homogénea), que van disminuyendo cuando la respuesta se hace más heterogénea (29). Esta homogeneidad de respuesta de una población susceptible concuerda con los resultados obtenidos para el fenitrotión en ninfas de primer y quinto estadio de $T$. dimidiata y ninfas de primer estadio de $T$. maculata. Sin embargo, no concuerda con la respuesta heterogénea detectada para los insecticidas $\beta$-cipermetrina y deltametrina en las dos especies. Probablemente esta heterogeneidad de respuesta en las especies estudiadas podría atribuirse al particular modo de acción de los piretroides en triatominos, en los que el efecto letal no tiene una muy clara manifestación y puede superponerse con un volteo prolongado, tal como se ha encontrado en $R$. prolixus (28). En $T$. dimidiata y T. maculata la intoxicación producida por piretroides se manifiesta por incoordinación, parálisis del tercer par de patas y convulsiones.

Al analizar los resultados obtenidos a través del cálculo del potencial insecticida en ninfas de primer estadio de T. dimidiata y $T$. maculata, se observa nuevamente una mayor efectividad insecticida de los piretroides frente al organofosforado. En las ninfas de quinto estadio de $T$. dimidiata, la toxicidad intrínseca de la deltametrina fue mayor comparada con la $\beta$ cipermetrina. Con las DL50 se observaron diferencias entre los piretroides y el fenitrotión; sin embargo, con la DL99 se encontró una mayor efectividad del organofosforado. Si bien este resultado hace pensar en una mayor efectividad del fosforado en condiciones de campo, es necesario plantear que la tolerancia de las ninfas $V$ de $T$. dimidiata a piretroides y fenitrotión representa un problema para las campañas de control de vectores de la enfermedad de Chagas en los países andinos y centroamericanos.

Recientemente, en Colombia se han reportado altas prevalencias de infestación intradomiciliarias y peridomiciliarias desde los primeros meses postratamiento por parte de adultos y ninfas de cuarto y quinto estadio de $T$. dimidiata, con un aumento creciente del número de triatominos capturados por mes de vigilancia en zonas rociadas con deltametrina (5) (Turriago BC, Pinto $\mathrm{N}$, Guhl F. Evaluación de deltametrina K-Othrine SC50 y K-Othrine WP 50 como alternativa de control de Triatoma dimidiata. Biomédica 2005;25:195).

Nuevos estudios con sinergistas podrían demostrar algún mecanismo que justifique esta tolerancia, lo cual ayudaría a mejorar las formulaciones de los insecticidas que se destinan al control de $T$. dimidiata.

Este estudio contribuye a la extensión del protocolo, evaluación de la actividad insecticida de los triatominos en Latinoamérica y permite el desarrollo de estudios en la siguiente etapa, es decir, comparar la línea de base de susceptibilidad con poblaciones de campo que hayan sido sometidas a control con los insecticidas probados. 
Todo esto en el marco de un seguimiento entomológico de las acciones de control químico en el país.

\section{Agradecimientos}

A Eduardo Zerba y al CIPEIN por su asesoría y revisión de este manuscrito.

\section{Conflicto de interés}

No hay conflicto de intereses.

\section{Financiación}

Este estudio fue financiado por UNDP/ World Bank/ WHO Special Programme for Research \& Training in Tropical Diseases (TDR), proyecto ID: 990163.

\section{Referencias}

1. World Health Organization. Infectious diseases home. Specific information: disease. 2004. [Consultado: abril 27 de 2005]. Disponible en: http://www.who.int/ $\mathrm{ctd} /$ chagas/burdens.htm.

2. Dujardin JP,Schofield CJ, Panzera F. Les vecteurs de la maladie de Chagas. Recherches taxonomiques, biologiques et génétiques. Bruxelles: Academie Royale des Sciences d'Outre Mer; 2000. p.162.

3. Aguilar M H, Abad Franch F, Racines VJ, Paucar CA. Epidemiology of Chagas disease in Ecuador. A brief review. Mem Inst Oswaldo Cruz 1999;94(Suppl. 1):387-93.

4. Schofield CJ. Challenges of Chagas disease vector control in Central America. Geneva: WHO;2000. p.36.

5. Angulo VM. Ensayo de estrategias de control y vigilancia de Triatoma dimidiata en Colombia. En: Guhl F, editor. Primer Taller Internacional Sobre Control de la Enfermedad de Chagas; Curso de Diagnóstico, Manejo y Tratamiento de la Enfermedad de Chagas. VI Reunión de la Iniciativa Andina para el control de la Enfermedad de Chagas. Bogotá D.C.: Ediciones Uniandes; 2005. p.91-102.

6. Guhl F, Angulo VM, Restrepo M, Nicholls S, Montoya R. Estado del arte de la enfermedad de Chagas en Colombia y estrategias de control. Biomédica 2003;23:31-4.

7. Molina JA, Gualdrón LE, Brochero HL, Olano VA, Barrios D, Guhl F. Distribución actual e importancia epidemiológica de las especies de triatominos (Reduviidae:Triatominae) en Colombia. Biomédica 2000;20:344-60.

8. Zeledón R, Montenegro VM, Zeledón O. Evidence of colonization of man-made ecotopes by Triatoma dimidiata (Latreille,1811) in Costa Rica. Mem Inst Oswaldo Cruz 2001;96:659-60.
9. Corredor A, Santacruz M, Paez S, Guatame LA. Distribución de los triatominos domiciliados en Colombia. Bogotá: Ministerio de Salud-Instituto Nacional de Salud; 1990 p.144.

10. Zerba EN. Susceptibility and resistance to insecticides of Chagas disease vectors. Medicina (B Aires) 1999;59:41-6.

11. Dias J, Schofield CJ. The evolution of Chagas disease (American trypanosomiasis) control after 90 years since Carlos Chagas discovery. Mem Inst Oswaldo Cruz 1999;94(Suppl. 1):103-21.

12. Guhl F, Vallejo GA. Interruption of Chagas disease transmission in the Andean countries: Colombia. Mem Inst Oswaldo Cruz 1999;94(Suppl. 1):413-5.

13. Angulo VM, Sandoval CM. Triatominos y Programa Nacional de Control en Colombia. Monitoreo de la resistencia a insecticidas en triatominos en América Latina, Buenos Aires: Fundación Mundo Sano, Red Latinoamericana de Control de Triatominos RELCOT; 2001. p.21-6.

14. Sánchez JF. Informe comportamiento del programa de Chagas, Cundinamarca años 2002-2005. Secretaría de Salud de Cundinamarca. En: Guhl F, editor. Primer Taller Internacional Sobre Control de la Enfermedad de Chagas. Curso de Diagnóstico, Manejo y Tratamiento de la Enfermedad de Chagas. VI Reunión de la Iniciativa Andina para el control de la Enfermedad de Chagas. Bogotá D.C: Ediciones Uniandes; 2005. p.135-40.

15. Nakagagua J, Cordón-Rosales C, Juárez J, Itzep C, Nonami T. Impact of residual spraying on Rhodnius prolixus and Triatoma dimidiata in the department of Zacapa in Guatemala. Mem Inst Oswaldo Cruz 2003;98:277-81.

16. Ceceré MC, Gurtler RE, Canale DM, Chuit R, Cohen JE. Effects of partial housing improvement and insecticide spraying on the reinfestation dynamics of Triatoma infestans in rural northwestern Argentina. Acta Trop 2002;84:101-16.

17. Tabarú Y, Monroy C, Rodas A, Mejia M, Rosales R. Chemical control of Triatoma dimidiata and Rhodnius prolixus (Reduviidae:Triatominae), the principal vectors of Chagas disease in Guatemala. Med Entomol Zool 1998;49:87-92.

18. Vassena CV, Piccollo MI, Zerba EN. Insecticide resistance in Brazilian Triatoma infestans and Venezuelan Rhodnius prolixus. Med Vet Entomol 2000;14:51-5.

19. Vassena CV, Piccollo MI. Monitoreo de resistencia a insecticidas en poblaciones de campo en Triatoma infestans y Rhodnius prolixus, insectos vectores de la enfermedad de Chagas. Boletín Electrónico Mensual del Servicio de Toxicología del Sanatorio de Niños de Rosario 2003:13. [Consultado: abril 5 de 2004]. Disponible en: http://www.sertox.com.ar/retel/n03/ 004.htm. 
20. Piccollo MI, Vassena C, Santo Orihuela P, Barrios S, Zaidemberg M, Zerba E. High resistance to pyrethroid insecticides associated with ineffective field treatments in Triatoma infestans (Hemiptera: Reduviidae) from Northern Argentina. J Med Entomol 2005; 42:637-42.

21. Oliveira Filho AM. Differences of susceptibility of five Triatomine species to pyrethroid insecticides -implications for Chagas disease vector control. Mem Inst Oswaldo Cruz 1999;94(Suppl. 1):425-8.

22. Fox I, Bayona IG. Toxicity of DDT, dieldrin, mlathion and fenthion to Rhodnius prolixus in the laboratory. Bull World Health Organ 1966;35:974-6.

23. Sandoval CM. Actividad insecticida del malatión y la deltametrina en una cepa colombiana de Rhodnius prolixus (Hemiptera:Rudiviidae). Monitoreo de la resistencia a insecticidas en triatominos en América Latina. Buenos Aires: Fundación Mundo Sano, Red Latinoamericana de Control de Triatominos RELCOT; 2001. p.27-33.
24. WHO. Protocolo de evaluación de efecto insecticida sobre triatominos. Acta Toxicol Arg 1994;2:29-32.

25. Zerba EN. Evolución del control químico de los insectos vectores de la enfermedad de Chagas. Anales de la Sociedad Científica Argentina 1997;227:35-9.

26. Alzogaray RA, Zerba EN. Third instar nymphs of Rhodnius prolixus exposed to $\alpha$-cyanopyrethroids: from hyperactivity to death. Arch Insect Biochem Physiol 2001;46:119-26.

27. Kostaropoulos I, Papadopoulos Al, Metaxakis A, Boukouvala E, Papadopoulou-Mourkidou E. Glutathione S-transferase in the defence against pyrethroids in insects. Insect Biochem Mol Biol 2001;31:3139

28. Fontán A, Zerba EN. Influence of the nutritional state of Triatoma infestans over the insecticidal activity of DDT. Comp Biochem Physiol C 1992;101:589-91.

29. Georghiou G. Insecticide resistance and prospects for its management. Residue Rev 1980;76:131-45. 